\title{
Ellis van Creveld: reporte de caso
}

\author{
M. Cruz-Perea • D. Luna • J. Ramírez-Cheyne •W. Saldarriaga • C. Isaza
}

\section{ABSTRACT \\ Ellis van Creveld. Case report}

Introduction: Ellis-van Creveld (EVC) (OMIM \# 225500) syndrome is a rare skeletal dysplasia disorder transmitted by autosomal recessive inheritance. The diagnosis is made based on phenotypic characteristics such as chondrodysplasia, heart defects and polydactyly. The prognosis depends mainly on the severity of the disease, diagnosis and comprehensive management of the condition. Objective: To describe a patient diagnosed with EVC syndrome. Case report: Newborn diagnosed with EVC syndrome who presented dysmorphic facies, shortened long bones, rhizomelic shortening, small hands, brachydactyly, single transverse palmar crease, postaxial polydactyly in the upper limbs, bilateral preaxial polysyndactyly in lower limbs and hypoplastic nails, complex heart defects and narrow thorax. The evolution was unfavorable; the patient died 8 weeks after birth from complications due to heart defects. Conclusions: EVC syndrome is rare and unknown; therefore, it is important to spread its characteristics within the pediatric community, emphasizing that it affects multiple organ systems and requires a multidisciplinary approach to treat individually each patient, to provide genetic and reproductive counseling to couples and to give information regarding child development expectations.

(Key words: Ellis-van Creveld syndrome, Chondroectodermal dysplasia, Polydactyly, Congenital anomalies). Rev Chil Pediatr 2014; 85 (5): 578-583

\section{RESUMEN}

Introducción: El síndrome Ellis-van Creveld (EVC) (OMIM \#225500) es una displasia esquelética rara de herencia autosómica recesiva, cuyo diagnóstico se realiza por sus características fenotípicas como la condrodis-

Recibido el 09 de enero de 2014, última versión aceptada el 03 de octubre de 2014.

Manuel Cruz-Perea, Dayhanna Luna Balcázar

Estudiante de Pregrado, Escuela de Medicina, Universidad del Valle. Cali, Colombia.

Julián Ramírez-Cheyne MD. ( $\square)$

Médico, Escuela de Ciencias Básicas Médicas, Universidad del Valle. Cali, Colombia.

E-mail: juracheyne@gmail.com

Wilmar Saldarriaga Gil MD., MSc.

Médico, Ginecólogo y obstetra, Magíster en Ciencias Básicas Medicas, Embriología y Genética. Profesor asociado, Escuela de Ciencias Básicas Médicas, Universidad del Valle. Cali, Colombia.

Carolina Isaza MD., MSc.

Médico, Magíster en Ciencias Básicas Médicas, Embriología y Genética. Profesor titular, Escuela de Ciencias Básicas Médicas, Universidad del Valle. Cali, Colombia. 
plasia, cardiopatía y polidactilia. El pronóstico depende fundamentalmente de la severidad de la cardiopatía, al igual que del diagnóstico y manejo integral oportunos. Objetivo: Caracterizar un paciente con diagnóstico clínico de Síndrome de EVC, cuya baja frecuencia dificulta el correcto diagnóstico en pediatría. Caso clínico: Recién nacido con facies dismórfica, extremidades con huesos largos cortos, acortamiento rizomélico, manos pequeñas, braquidactilia, pliegue palmar único, polidactilia post axial en miembros superiores, polisindactilia preaxial bilateral en miembros inferiores y uñas hipoplásicas, cardiopatía compleja y tórax estrecho, en el que se concluyó un diagnóstico clínico de EVC. La evolución fue desfavorable, falleciendo a las 8 semanas de nacimiento por complicaciones secundarias a la cardiopatía. Conclusiones: El síndrome de EVC es de baja frecuencia y poco conocido, por lo que es importante difundir sus características en la comunidad pediátrica, haciendo énfasis en que al afectar múltiples sistemas y órganos, requiere un manejo multidisciplinario con el objetivo de intervenir en la patología individualizando cada paciente; además de consejería genética y reproductiva a las parejas, e información de las expectativas del desarrollo del niño.

(Palabras clave: Síndrome Ellis-van Creveld, Displasia condroectodérmica, Polidactilia, Anomalías congénitas).

Rev Chil Pediatr 2014; 85 (5): 578-583

\section{Introducción}

El Síndrome Ellis-van Creveld (EVC) (OMIM \#225500) es una displasia condroectodérmica que se caracteriza por costillas cortas, polidactilia, retraso del crecimiento, defectos ectodérmicos y anomalías cardíacas; producidas por mutaciones en los genes $E V C$ y $E V C 2$ (Ellis van Creveld y Ellis van Creveld 2) localizados en 4 p16 y que tienen un patrón de herencia autosómica recesiva ${ }^{1}$.

Las mutaciones en $E V C$ se describieron inicialmente en las genealogías Amish y en Brasil, aunque estas sólo representan una pequeña proporción de la totalidad de afectados ${ }^{2}$. Posteriormente, se describió la mutación en el gen $E V C 2$ en un niño Asquenazí que presentaba este síndrome ${ }^{3}$. Los pacientes con mutaciones en $E V C$ o $E V C 2$ son fenotípicamente indistinguibles ${ }^{4}$.

Es una enfermedad poco frecuente, de prevalencia estimada en 7 por cada 1.000 .000 de habitantes en población general ${ }^{5}$, sin embargo, es conocido que los trastornos recesivos infrecuentes pueden tener frecuencias elevadas en grupos genéticamente aislados o con efecto fundador, como es el caso de la frecuencia de EVC en el grupo etno-religioso Amish ${ }^{6}$. Uno de los mayores asentamientos de este grupo se ubica en el condado de Lancaster, Pennsylvania, y presenta una prevalencia de EVC estimada en 5 por 1.000 nacidos vivos, siendo esta la más alta conocida ${ }^{7}$. La frecuencia de portadores en esta población puede ser hasta de un $13 \% \%^{8}$. Para este reporte se atendieron las respectivas consideraciones éticas, dentro de las que realizó un consentimiento informado a los padres del paciente.

\section{Objetivo}

Caracterizar un caso de paciente con diagnóstico clínico de Síndrome de EVC. Se hace la discusión del caso con una breve revisión de la literatura, dando información a la comunidad pediátrica de un síndrome poco conocido.

\section{Caso clínico}

Recién nacido de sexo masculino, cesárea a las 38 semanas de edad gestacional debido a una cardiopatía compleja; peso de nacimiento $2.860 \mathrm{~g}$ (p15), longitud $44 \mathrm{~cm}$ (p3), perímetro cefálico $33 \mathrm{~cm}$ (p 3 ). Padres no consanguíneos sin antecedentes personales ni familiares de importancia, madre primigesta de 19 años.

A las 12 semanas de gestación se encontró translucencia nucal mayor de p95, por lo que se realizó un cariotipo en biopsia de vellosidades coriales que mostró un resultado 46, XY; a las 18 semanas una ecografía evidenció cardiopatía compleja, con un canal aurículoventricular completo, mientras que en una eco- 


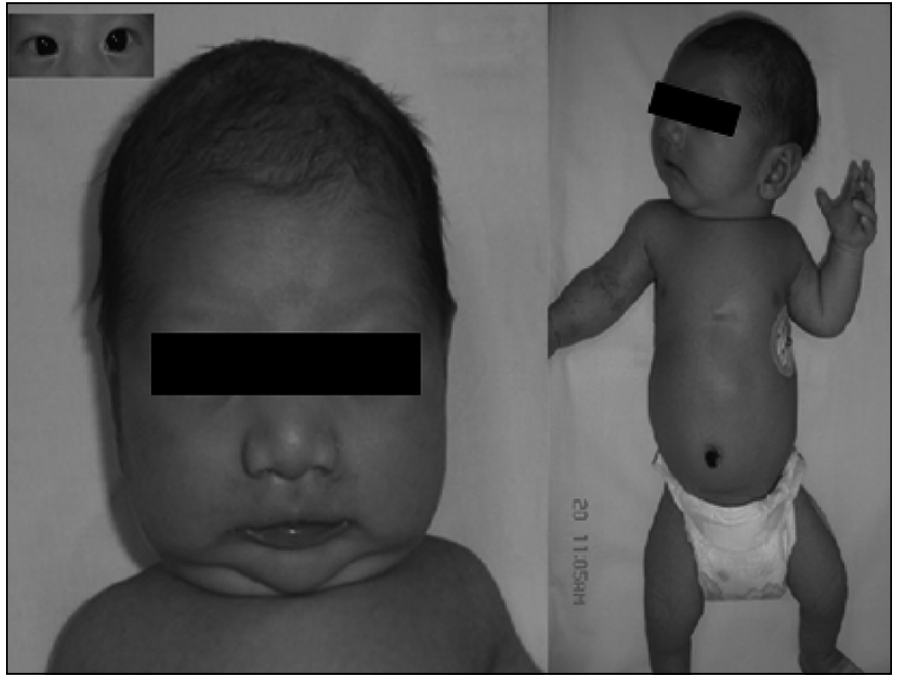

Figura 1. Foto de acercamiento facial y vista de cabeza, tronco y extremidades parcialmente. Se observa orejas de implantación baja con rotación hacia atrás y puente nasal deprimido. Además acortamiento de todas las extremidades al comparar con el tronco; tórax estrecho, mostrando desproporción con el abdomen.

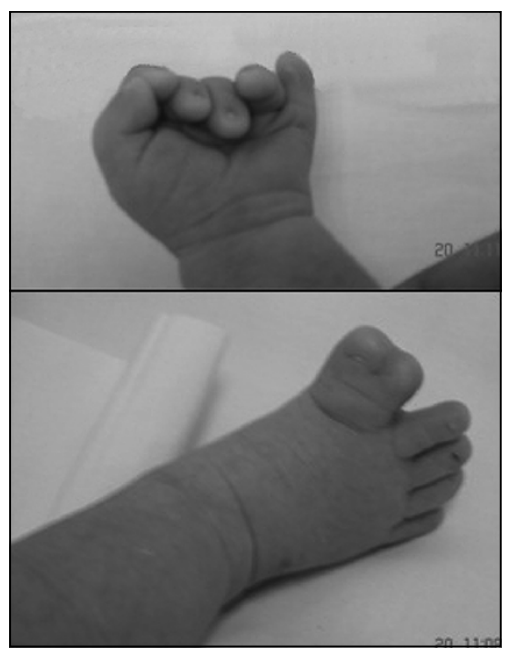

Figura 2: Foto de mano y pie, se observa polidactilia post axial en mano y preaxial en miembro inferior; además uñas hipoplásicas.

grafía de tercer trimestre los huesos largos se encontraban en $\mathrm{p} 5$.

Al nacimiento fue hospitalizado en unidad de cuidados intensivos neonatal. Evaluado por genetista clínico, quien al examen dismorfológico describió: microcefalia, orejas de implantación baja con rotación hacia atrás, puente nasal deprimido, pliegue epicántico interno, estrabismo convergente, en boca labio superior corto unido por frenillos al reborde alveolar, extremidades con huesos largos cortos, rizo- melia (figura 1), manos pequeñas, braquidactilia, pliegue palmar único, polidactilia post axial en miembros superiores, polisindactilia preaxial bilateral en miembros inferiores $\mathrm{y}$ uñas hipoplásicas (figura 2).

El ecocardiograma mostró canal aurículoventricular, estenosis aórtica, hipoplasia del arco aórtico, ductus arterioso e hipoplasia leve de arteria pulmonar derecha, también se realizó ecografía transfontanelar y renal que resultaron normales; radiografías de miembros inferiores mostraron fémur y la tibia cortos con adecuada osificación, en radiografía toracoabdominal se observó un tórax estrecho al comparar con el abdomen y una silueta cardíaca aumentada de tamaño al comparar con el tórax (figura 3); en la columna no se observaron alteraciones vertebrales (figura 3 ).

La suma de hallazgos permitió diagnosticar el síndrome de Ellis Van Creveld (tabla 1). El paciente tuvo una evolución desfavorable falleciendo a las 8 semanas de nacido, por problemas secundarios a su cardiopatía compleja.

\section{Discusión}

Desde la descripción del síndrome de EVC en el año 1940, con el nombre de Displasia condroectodérmica, se han reportado en la literatura aproximadamente $150 \operatorname{casos}^{1}$. El caso 


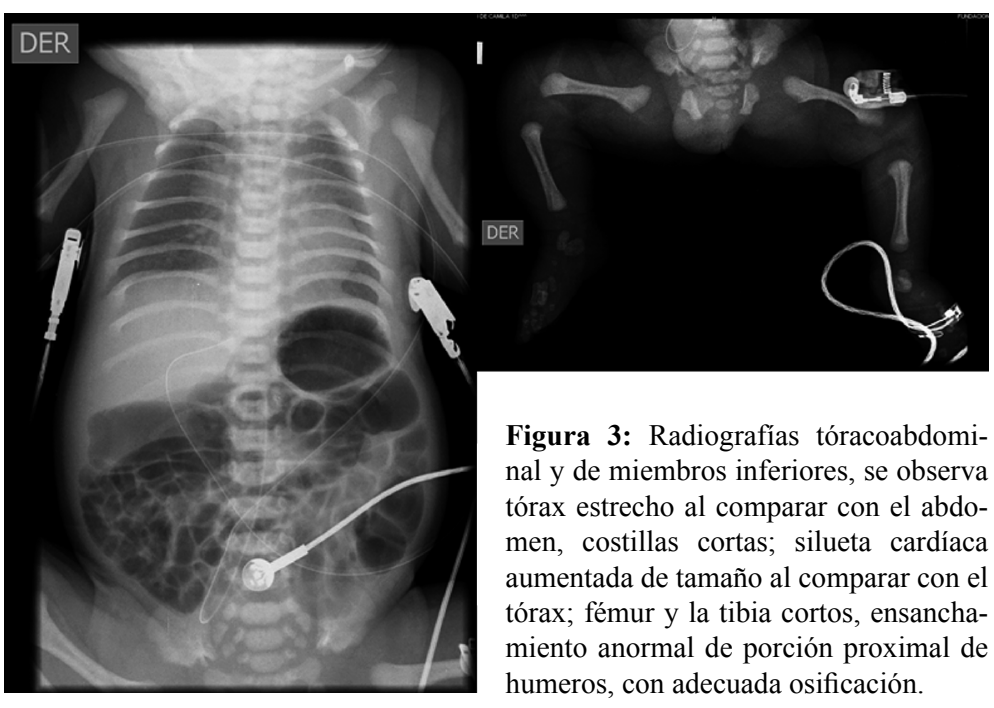

Tabla 1. Se comparan características fenotípicas reportadas (OMIM 225500) para el síndrome de Ellis van Creveld (EVC) y las encontradas en el paciente

\begin{tabular}{|c|c|}
\hline Características reportadas en la literatura & Características presentadas por el paciente \\
\hline Talla baja con extremidades cortas al nacimiento & $\mathrm{Si}$ \\
\hline Normocefalia & No (Microcefalia) \\
\hline Facies normales (con excepción del labio superior) & Pliegue epicantico interno, puente nasal deprimido \\
\hline Anomalías del labio superior & Si (Labio superior corto) \\
\hline Labio fisurado & No \\
\hline Defectos de cresta alveolar & Si (sinequia labio-encía) \\
\hline Dientes neonatales & No \\
\hline Hipodontia, Erupción retrasada & No aplica \\
\hline Defecto septal atrial, Atrio único & Si \\
\hline Canal aurículoventricular & Si \\
\hline Defecto septal ventricular & Si \\
\hline Ductus arterioso persistente & Si \\
\hline Otros defectos cardíacos & Si \\
\hline Tórax estrecho & Si \\
\hline Pectus carinatum & No \\
\hline Costillas cortas, poco desarrolladas & Si \\
\hline Anomalías génito urinarias & No \\
\hline Rizomelia & Si \\
\hline Braquidactilia & Si \\
\hline Polidactilia en manos y pies & Si \\
\hline Epífisis en cono de las falanges 2 a 5 de las manos & No evaluado \\
\hline Pie equinovaro & No \\
\hline Sindactilia en dedos de pies & Si \\
\hline Displasia de uñas & Si \\
\hline Déficit cognitivo & No aplica \\
\hline
\end{tabular}


aquí presentado sería el segundo reportado en Colombia9.

Este síndrome tiene una expresión fenotípica variable, observable en el período fetal a través de ecografía obstétrica, lo cual permite sospechar prenatalmente su presencia ${ }^{8,10}$, al encontrar hallazgos de aumento de la translucencia nucal y cardiopatía ${ }^{11}$, como ocurrió en el caso reportado.

Los pacientes con EVC presentan talla menor al percentil 3, con extremidades desproporcionadas con respecto del tronco, anomalías esqueléticas, polidactilia principalmente en manos, generalmente bilateral, ocasionalmente en los pies; cardiopatías como: aurícula única, defectos de la válvula mitral y tricúspide, ducto arterioso persistente, comunicación interventricular, comunicación interauricular e hipoplasia cardíaca izquierda ${ }^{12}$; displasia de uñas, cabello quebradizo y erupción temprana de dientes ${ }^{13}$. Su perímetro cefálico suele ser normal y usualmente no se presentan anomalías del sistema nervioso central por lo que el desarrollo cognitivo es normal ${ }^{4}$. El paciente presentado mostró todas las características del síndrome de EVC al nacimiento excepto la erupción temprana de dientes.

La condrodistrofia es un rasgo característico y se manifiesta con retardo en la maduración ósea, fusión de los huesos del carpo y metacarpo, depresión proximal y lateral de la tibia, luxación congénita de rótula, cubito valgo, hipoplasia cubital, genu valgo, clinodactilia del quinto dedo y alteración del modelado óseo de los metacarpianos y falanges. El tórax presenta frecuentemente costillas cortas y estrechas, con pectus excavatum y lordosis lumbar aumentada ${ }^{9,12}$.

Hasta en un $93 \%$ de los casos se presenta displasia ectodérmica hidrótica caracterizada por uñas friables, distróficas, hipoplásicas o incluso completamente ausentes; el cabello puede ser escaso o quebradizo; y las anomalías dentarias incluyen presencia neonatal de dientes, anodoncia parcial, hipoplasia del esmalte que se manifiesta con dientes pequeños anormalmente formados, mal oclusión o retraso en la erupción ${ }^{1,8,9}$.

Pueden encontrarse pacientes con EVC que presenten agenesia o displasia renal, megauré- ter, epi o hipospadias, en un 20\% de los casos ${ }^{1}$; en menor proporción estrabismo, malformaciones pulmonares, de la pared torácica ${ }^{1,7}, \mathrm{y}$ sólo un caso en la literatura reporta alteraciones hematológicas (leucemia mieloblástica perinatal $)^{13}$.

El síndrome EVC hace parte un grupo denominado como "síndromes de costillas cortas-polidactilia", donde también se encuentran el síndrome de Saldino-Noonan (tipo I), el síndrome de Majewski (tipo II), el síndrome de Verma-Naumoff (tipo III), el síndrome de Beemer-Langer (tipo IV) y distrofia de Jeune ${ }^{10}$.

Las características fenotípicas comunes de estos síndromes pueden dificultar su diferenciación, aunque existen indicios que permiten aclarar el diagnóstico: paladar hendido en Majewski; onfalocele y fémur curveado en Beemer-Langer ${ }^{10}$; distrofia torácica, reducción de las extremidades y displasia ósea generalizada en Jeune ${ }^{1}$; prevalencia mayor de cardiopatías, condrodisplasia y polidactilia postaxial en $\mathrm{EVC}^{1}$. Además en el paciente reportado se diferenció del Síndrome de Saldino-Noonan, por no observarse en las radiografías las costillas deformadas.

Se debe sospechar el Síndrome de EVC en casos de cardiopatías complejas principalmente aurícula única, facies dismórficas, tórax estrecho, acortamiento rizomélico, polidactilia; en estos casos se deben realizar radiografías del tórax observando detenidamente las costillas; y de extremidades buscando condrodistrofias; ecografía renal con el objeto de descartar agenesia y alteraciones de la vía urinaria; además se deben descartar alteraciones cromosómicas numéricas o estructurales con cariotipo con bandeo $\mathrm{G}$, e idealmente realizar la prueba molecular para $E V C$ y $E V C 2$, teniendo en cuenta que en un tercio de los pacientes estas pueden no mostrar alteraciones ${ }^{14}$.

El manejo integral de un paciente con EVC se debe realizar por un grupo multidisciplinario, idealmente desde la etapa prenatal, identificando características fenotípicas sugestivas del síndrome, permitiendo preparar a la familia y particularmente al equipo de atención neonatal, para tratar adecuada y oportunamente los problemas respiratorios y cardíacos, ya que si estos se superan mejora el pronóstico. El desa- 
rrollo motor de los pacientes que logran superar la etapa neonatal depende de las alteraciones y deformidades óseas que ellos presenten. Además se debe realizar consejería genética y reproductiva a los padres dentro de dicho manejo integral; la probabilidad de recurrencia del evento es $25 \%$ en cada embarazo.

\section{Conclusión}

El síndrome de EVC es un síndrome de baja frecuencia, poco conocido, por lo que es importante difundir sus características en la comunidad pediátrica. Debido a que en este síndrome se afectan múltiples sistemas y órganos, requiere un manejo multidisciplinario que incluye un genetista clínico, con el objetivo de intervenir adecuadamente en la patología, individualizando cada paciente; además de brindar consejería genética y reproductiva a las parejas y dar adecuada información de las expectativas del desarrollo del niño.

Potenciales conflictos de interés: Este trabajo cumple con los requisitos sobre consentimiento/asentimiento informado, comité de ética, financiamiento, estudios animales y sobre la ausencia de conflictos de intereses según corresponda.

\section{Referencias}

1.- Baujat G, Le Merrer M: Ellis-van Creveld syndrome. Orphanet J Rare Dis 2007; 2: 27.

2.- Ruiz-Pérez VL, Blair HJ, Rodríguez-Andrés ME, Blanco MJ, Wilson A, Liu YN: Evc is a positive mediator of Ihh-regulated bone growth that localises at the base of chondrocyte cilia. Development 2007; 134 (16): 2903 12 .
3.- Galdzicka M, Patnala S, Hirshman MG, Cai JF, Nitowsky H, Egeland JA: A new gene, EVC2, is mutated in Ellis-van Creveld syndrome. Mol Genet Metab 2002; 77 (4): 291-5.

4.- Ruiz-Pérez VL, Ide SE, Strom TM, et al: Mutations in a new gene in Ellis-van Creveld syndrome and Weyers acrodental dysostosis. Nature Genet 2000; 24: 283-6.

5.- Zeng Z, Kun B, Jin-Wei H, Wen-Zhen F, Chang-Qing Z, Zhen-Lin Z: Identification of one novel mutation in the EVC2 gene in a Chinese family with Ellis-van Creveld syndrome, Gene 2012; 511: 380-2.

6.- Thompson MW, McLnnes RR, Willard HF: Genética en Medicina. 7a ed. Barcelona, Masson, S. A. 2008.

7.- McKusick VA: Ellis-van Creveld syndrome and the Amish. Nat Genet. 2000; 24 (3): 203-4.

8.- Chen H: Ellis van Creveld Syndrome. Medscape. com. http://emedicine.medscape.com/article/943684overview\#showall . Updated: Aug 11, 2011.

9.- Estrada T, Montoya J, Cortés-Yepes H: Diagnóstico prenatal del Síndrome Ellis Van Creveld: reporte de caso y revisión de la literatura. Revista Colombiana de Obstetricia y Ginecología 2011; 62 (3): 250-4.

10.- Elçioglu NH, Hall CM: Diagnostic dilemmas in the short rib-polydactyly syndrome group. Am J Med Genet 2002; 111: 392-400.

11.- Venkat-Raman N, Sebire NJ, Murphy KW, Carvalho JS, Hall CM: Increased first-trimester fetal nucal translucency thickness in association with chondroectodermal dysplasia (Ellis-van Creveld syndrome). Ultrasound Obstet Gynecol 2005; 25: 412-4.

12.- Slavkin HC: What the future holds for ectodermal dysplasias: future research and treatment directions. Am J Med Genet A 2009; 149: 2071-4.

13.- Scurlock D, Ostler D, Nguyen A, Wahed A: Ellis-van Creveld syndrome and dyserythropoiesis. Arch Pathol Lab Med 2005; 129: 680-2.

14.- Tompson SW, Ruiz-Pérez VL, Blair HJ, et al: Sequencing EVC and EVC2 identifies mutations in two-thirds of Ellis-van Creveld syndrome patients. Hum Genet 2007; 120 (5): 663-70. 